\title{
Metastatic inguinal lymph nodes with two different histological types in a case of carcinoma of unknown primary site
}

\author{
Mukur Dipi Ray, Shivam Vatsal, Sunil Kumar \\ Department of Surgical Oncology, Dr. BRA Institute Rotary Cancer Hospital, All India Institute of Medical Sciences, New Delhi 110029, India.
}

Correspondence to: Dr. Sunil Kumar, Department of Surgical Oncology, Dr. BRA Institute Rotary Cancer Hospital, All India Institute of Medical Sciences, Ansari Nagar, New Delhi 110029, India. E-mail: dr_sunilk@hotmail.com

\section{A B S T R A C T}

Cancer of unknown primary site is a group of uncommon cancers where patients present with metastatic disease and the primary site is not identified, even after a complete workup to establish the diagnosis. Inguinal metastasis with unknown primary is even more uncommon, and histological type is the most important guiding factor to look for the primary. This report describes the rare situation of inguinal metastasis with an unknown primary site where a combination of squamous and transitional cell carcinoma was found on final histopathology. It highlights the importance of multimodality approach including an aggressive surgical resection combined with adjuvant radiation therapy to achieve an optimal outcome.

Key words: Carcinoma of unknown primary site, inguinal metastasis, squamous cell carcinoma, transitional cell carcinoma

\section{Introduction}

Cancer of unknown primary site (CUPS) is a clinical syndrome that is considered in patients where, even after extensive standard clinical, pathological and radiological evaluation, the primary site cannot be identified. Patients with CUPS account for $0.5-4 \%$ of all cancers diagnosed. ${ }^{[1]}$ Within this heterogeneous group, there is a wide variation of clinical presentations and histological types. Most present as a metastatic disease, which is often difficult to categorize using histology alone. Immunohistochemistry (IHC) is helpful in separating carcinomas from a neoplasm of other lineages. CUPS is more common in the head and neck and axillary regions, and inguinal involvement accounts for $<5 \%$ of cases. ${ }^{[2,3]}$ Metastatic inguinal lymphadenopathy mainly originates from the genitalia and anorectal areas. In this case report, we describe an uncommon case of two different histological types of metastases in inguinal nodes with unknown primary sites.

\section{Case Report}

A 49-year-old male patient, a farmer, presented in October 2012 to the surgical oncology clinic with swelling in the right groin crease for 2 years, which had been increasing progressively in size and subsequently became ulcerated. On examination the mass was hard, irregular in shape

\begin{tabular}{|l|l|}
\hline \multicolumn{2}{|c|}{ Access this article online } \\
\hline Quick Response Code: & Website: \\
\hline & www.jcmtjournal.com \\
\cline { 2 - 2 } & \\
\hline
\end{tabular}

due to the conglomeration of inguinal lymph nodes, about $5 \mathrm{~cm}$ in diameter, fixed to the skin and deeper structures, and superficially ulcerated [Figure 1]. Bilateral hydroceles were also present. No other enlarged lymph nodes in other regions were palpable. Per rectal examination and clinical evaluation, the genitals were normal. Fine-needle aspiration cytology of the node was suggestive of squamous cell carcinoma. For better categorization, a biopsy was performed, which was suggestive of poorly differentiated squamous cell carcinoma. On contrast-enhanced computed tomography (CT) scan, an ill-defined mass lesion of $5 \mathrm{~cm} \times 4 \mathrm{~cm}$ was noted over right inguinal region encasing the femoral vein and having $180^{\circ}$ contact with the femoral artery [Figure 2]. Right external iliac and obturator nodes were also enlarged. The remainder of the abdomen and chest were normal. Positron emission tomography (PET) scan showed increased tracer uptake in right inguinal, external iliac, and obturator nodes, but a primary site could not be visualized. Ultrasound evaluation of the testes was normal. Upper and lower gastrointestinal endoscopy were normal. Serum carcinoembryonic antigen, CA19-9, alpha-fetoprotein, prostate specific antigen, and beta human chorionic gonadotropin were within normal range.

With no primary site of cancer identified, the patient was taken for a right ilioinguinal lymph node dissection. The nodal mass, along with the encased segment of the femoral vein, was resected, and an autologous internal jugular vein graft was placed [Figures 3 and 4]. Lymph nodal clearance up to the aortic bifurcation was done. The postoperative period was uneventful. Histopathology was suggestive of squamous cell carcinoma with islands of transitional cells in interposed [Figure 5]. IHC stainings for CK20, CK5, CK6, and CK7 were negative. In view of the transitional cell elements, cystoscopy, 


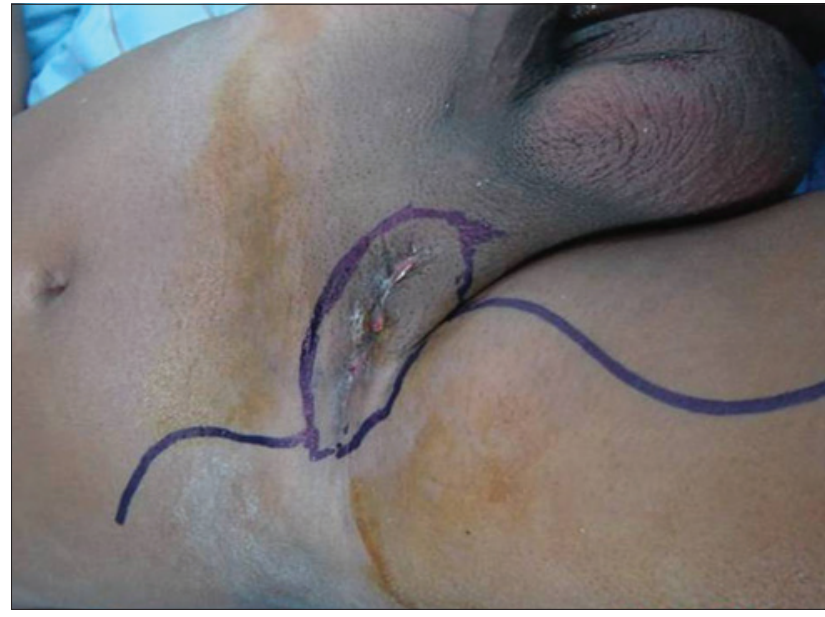

Figure 1: Clinical appearance of right inguinal nodes

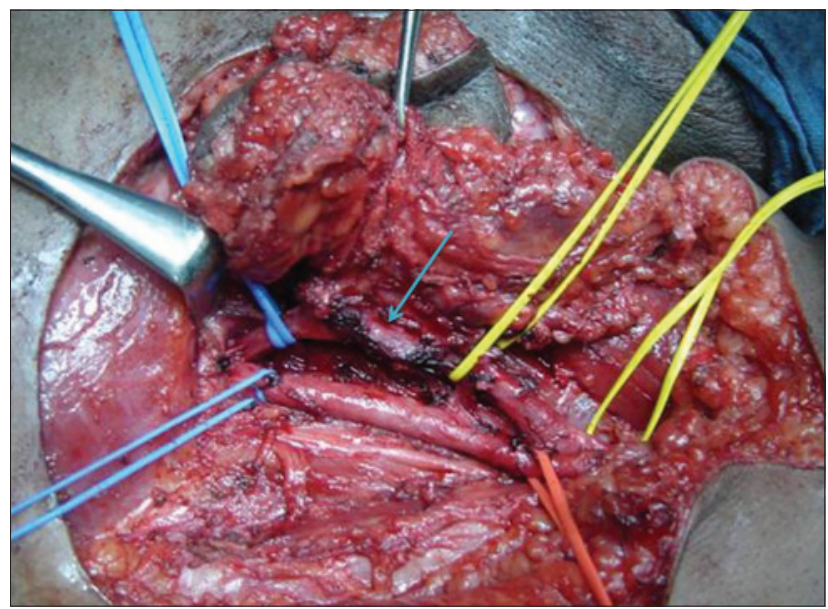

Figure 3: Intra-operative picture of nodal mass infiltrating femoral vein (site of infiltration marked with arrow)

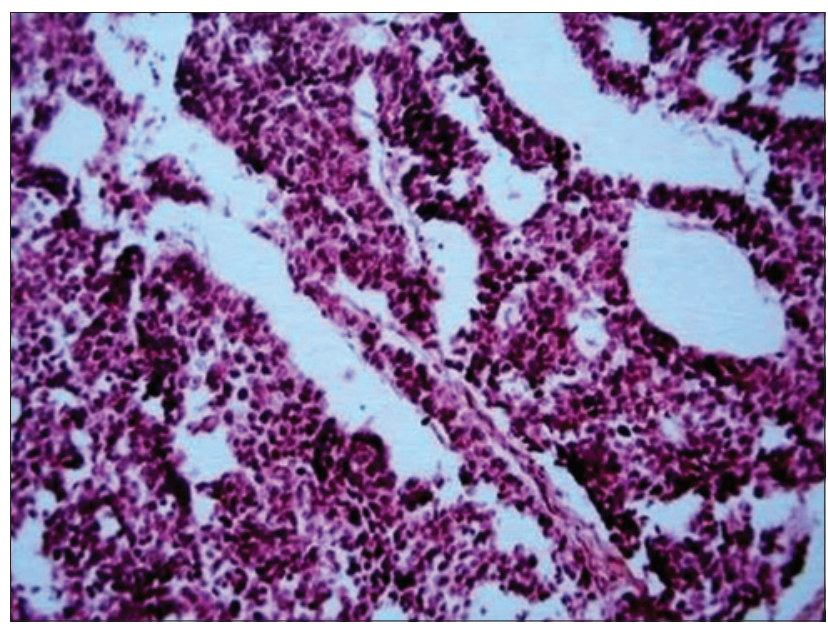

Figure 5: Histopathology picture from lymph node showing squamous cell carcinoma with areas of transitional cell carcinoma. $(\times 40)$

and urine analysis were also done, both of which were normal. Adjuvant radiotherapy to the bilateral inguinal, pelvic, and para-aortic regions with a dose of $55 \mathrm{~Gy} / 25$ fractions over 5 weeks was given. The patient tolerated the radiotherapy with minimal complications and was

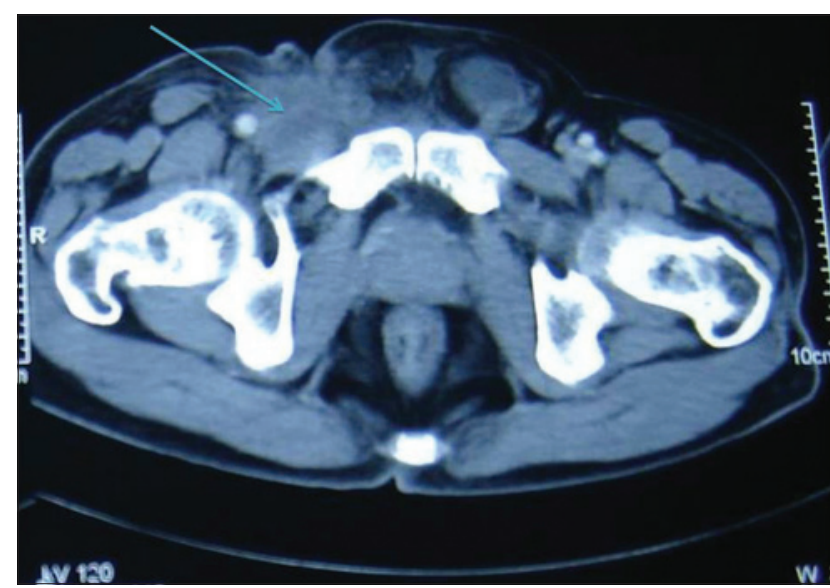

Figure 2: Contrast-enhanced computed tomography of pelvis showing righ inguinal nodal mass (marked with arrow) infiltrating femoral vein

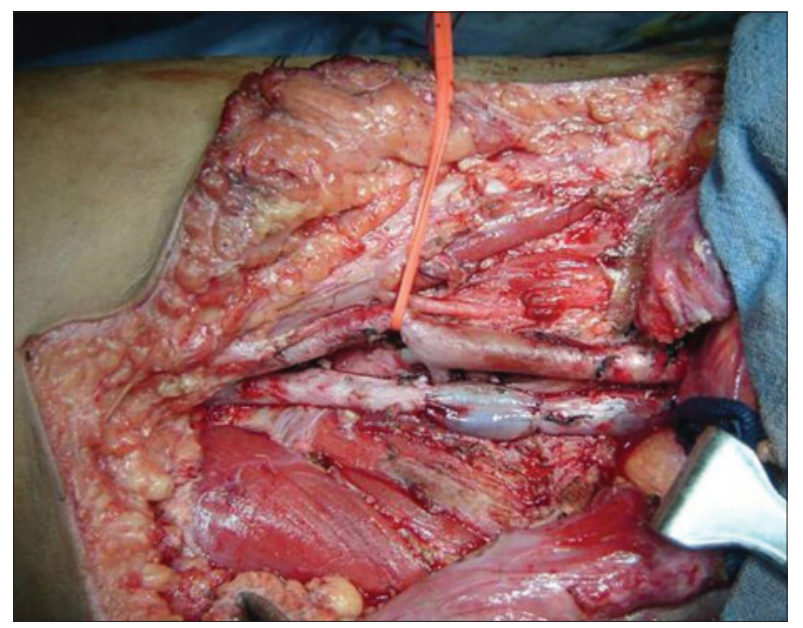

Figure 4: Reconstructed femoral vein with interposition graft from internal jugular vein

doing well in the last follow-up one year post-surgery. All investigations and tumor markers repeated at the last follow-up in December 2014 were normal.

\section{Discussion}

The inguinal area is a relatively uncommon metastatic site of CUPS. ${ }^{[4]}$ There has been a wide variety of primary sites from where inguinal nodal metastasis has been reported. These include some sites, which are quite distant from the pelvis (nasopharynx, breast, tracheobronchial tree, salivary glands, orbit) but most originate in the pelvis, genitalia or lower limb..$^{[2,5,6]}$ In one of the largest series, involving more than 2,000 patients with inguinal nodal metastasis, the primary site could not be identified in $22(1 \%)$, even after a significant period of follow-up. ${ }^{[2]}$ In the present case, even after extensive attempts to find the primary site, the site could not be determined. The final histopathology of this patient showed a mixed picture of squamous and transitional cell carcinoma, with the former predominating. A literature search revealed no such report of two different histological types of tumors, squamous and transitional, in the same patient at the 
same site, from an unknown primary site, although there are reports of mixed squamous and adenocarcinomas. ${ }^{[7]}$

The clinical investigative approach toward CUPS patients is mainly directed according to the histopathology, and every attempt should be made to obtain a good tissue sample for detailed IHC analysis. Investigations should involve a multi-modality approach. The role of PET scan is yet to be established but has the potential to modify the treatment in some patients whose tumor was localized with CT. ${ }^{[8]}$ As early as 1979 , it was emphasized that the analysis of tissue samples should help to eliminate the need for undirected investigations screening for the primary site. ${ }^{[9]}$ Since then, there have been significant advances in the molecular analysis of tumors, and so the incidence of CUPS has decreased. ${ }^{[10]}$

Since CUPS in the inguinal region is rare, there is a paucity of literature on the management of such patients, and no clear guidelines are described. The mainstay of treatment is surgery, with complete surgical excision through systematic lymph nodal dissection being mandatory. Aggressive surgical treatment including vascular resection and reconstruction with grafting may be required to achieve tumor-free margins, as was the situation in this case. Although role of postoperative radiotherapy is not clearly defined, it is thought that, in the presence of extensive nodal involvement and/or extranodal spread of tumor, postoperative radiotherapy should be used as it would be with any known primary site with squamous cell carcinoma. A review article indicates that surgery with adjuvant irradiation was the preferred treatment for inguinal metastasis with the unknown primary site. ${ }^{[11]}$

A diligent follow-up is required for these patients. In one case report described an occult carcinoma of the penis manifested 3 years after treatment of inguinal nodal metastasis. ${ }^{[12]}$ According to the authors, circumcision and random biopsy of glans should be a routine of such patients. The patient in the present case was also followed up clinically, radiologically, and with cystoscopy in view of the presence of transitional cell carcinoma.

Carcinoma of unknown primary with inguinal metastasis is a rare entity. Investigations should be directed to identify the primary site according to histopathology. Although there are no clear guidelines for the management of such patients, treatment should be multimodal, including aggressive surgical resection, and postoperative radiotherapy. The possible role of chemotherapy is unknown. A diligent follow-up is a must. In the future, molecular studies may increase our ability to distinguish subtypes of CUSP and treat them differentially.

\section{References}

1. Greco FA, Hainsworth JD. Introduction: Unknown primary cancer. Semin Oncol 2009;36:6-7.

2. Zaren HA, Copeland EM 3rd. Inguinal node metastases. Cancer 1978;41:919-23.

3. Guarischi A, Keane TJ, Elhakim T. Metastatic inguinal nodes from an unknown primary neoplasm. A review of 56 cases. Cancer 1987;59:572-7.

4. Pavlidis N, Pentheroudakis G. Cancer of unknown primary site. Lancet 2012;379:1428-35.

5. Wallack MK, Reynolds B. Cancer to the inguinal nodes from an unknown primary site. J Surg Oncol 1981;17:39-43.

6. Massard C, Loriot Y, Fizazi K. Carcinomas of an unknown primary origin - diagnosis and treatment. Nat Rev Clin Oncol 2011;8:701-10.

7. Takeuchi T, Yasui T, Izeki M, Komune S. Adenosquamous carcinoma of unknown primary origin: a case report and literature review. J Laryngol Otol 2015;129 Suppl 2:S91-4.

8. Alberini JL, Belhocine T, Hustinx R, Daenen F, Rigo P. Whole-body positron emission tomography using fluorodeoxyglucose in patients with metastases of unknown primary tumours (CUP syndrome). Nucl Med Commun 2003;24:1081-6.

9. Osteen RT, Kopf G, Wilson RE. In pursuit of the unknown primary. Am J Surg 1978;135:494-7.

10. Oien KA, Dennis JL. Diagnostic work-up of carcinoma of unknown primary: from immunohistochemistry to molecular profiling. Ann Oncol 2012;23 Suppl 10:x271-7.

11. Pavlidis N, Briasoulis E, Hainsworth J, Greco FA. Diagnostic and therapeutic management of cancer of an unknown primary. Eur J Cancer 2003;39:1990-2005.

12. Sinha M, Katema M, Malata CM. Squamous cell carcinoma of penis presenting as groin metastasis 3 years before the primary. J Plast Reconstr Aesthet Surg 2006;59:547-9.

How to cite this article: Ray MD, Vatsal S, Kumar S. Metastatic inguinal lymph nodes with two different histological types in a case of carcinoma of unknown primary site. J Cancer Metastasis Treat 2015;1:101-3.

Received: 21-01-2015; Accepted: 08-05-2015.

Source of Support: Nil, Conflict of Interest: None declared. 